\title{
"Und ich hab damals für dich ge-Voted..." Online-Kommentarforen zwischen Text, Diskurs und Gemeinschaft
}

\author{
„And I have voted for zou back then..." Online Comments Sections Between \\ Text, Discourse and Community
}

\begin{abstract}
ZUSAMMENFASSUNG
Im Artikel wird am Beispiel von Leser*innenkommentaren im Schweizer Online-Newsportal watson.ch der Frage nachgegangen, inwiefern sich bei Online-Communities Merkmale von traditionaler Vergemeinschaftung (sensu Max Weber) nachzeichnen lassen und ob sich das Konzept der Diskursgemeinschaft(en) damit in Verbindung bringen lässt. Dafür wird der Begriff der Diskursgemeinschaft zunächst im widersprüchlichen Spannungsfeld zwischen sprach- und sozialwissenschaftlichen Gemeinschaftsverständnissen verortet. Anschliessend wird anhand ausgewählter Kommentare untersucht, wie sich klassische Vergemeinschaftungsmerkmale in Kommentarforen auf empirischer Ebene manifestieren. Abschliessend wird ein Lösungsvorschlag skizziert, wie die Systemtheorie nach Niklas Luhmann die Aporien zwischen verschiedenen on- und offline Vergemeinschaftungsformen überbrücken und damit einen integrativen Rahmen für die konfligierenden Gemeinschaftsverständnisse der klassischen Soziologie und der Diskursanalyse anbieten kann.

Schlüsselwörter: Textlinguistik, Systemtheorie, Diskursanalyse, Online-Vergemeinschaftung, Leserkommentare
\end{abstract}

\begin{abstract}
Using examples from comment sections in the Swiss online news site watson.ch, we investigate the question whether such online reader communities show traces of communal relationships (in the sense of Max Weber) and if such an understanding is compatible with the concept of discourse communities. To this end, we first outline the conflicting theoretical assumptions that linguistic and sociological understandings of communities imply for the concept of discourse communities. Afterwards, we use selected online reader comments to show how traditional features of community formation can be detected empirically in the comment section. To conclude, we argue that Niklas Luhmann's systems theory can serve as a framework to integrate different types of community formation processes on an empirical and a theoretical level.
\end{abstract}

Keywords: online community, reader comments, online discourse analysis, systems theory, text linguistics

Marcel Naef, Deutsches Seminar, Universität Zürich, Schönberggasse 9, 8001 Zürich, marcel.naef@ds.uzh.ch, https://orcid.org/0000-0003-2520-0300 


\section{Einleitung}

In der diskurslinguistischen Erforschung des World Wide Web spielen Kommunikate von Online-Communities eine zunehmend bedeutendere Rolle, wenn es um die Aufdeckung diskursiver Strukturen und die Rekonstruktion der darin agierenden Diskursgemeinschaft(en) geht. Das Verhältnis zwischen Online-Community und Diskursgemeinschaft wurde bisher allerdings nie zum Gegenstand theoretischer oder empirischer Erörterungen. Es bleibt unklar, was diese beiden Gebilde genau zu Gemeinschaften bzw. Communities macht und welche theoretischen Konzeptionen von Sozialität daran anschlussfähig wären. Es sind offene Fragen, ob die beiden Begriffe deckungsgleich sind, ob eine Online-Community jeweils mehrere Diskursgemeinschaften beinhaltet, ob eine Diskursgemeinschaft sich aus mehreren Communities zusammensetzt oder ob sie sich wechselseitig durchdringen. Diese begrifflichen Unschärfen sind auf den ersten Blick insofern nachvollziehbar, als es sich bei Online-Communities um eine ethnokategorische Bestimmung, bei Diskursgemeinschaften hingegen um eine fachsprachliche Konstruktion handelt. Der eigentliche Hauptgrund für die begrifflichen Unschärfen dürfte aber sein, dass sich Online-Communities und Diskursgemeinschaften aufgrund ihrer spezifischen konstitutiven Ausprägungen nicht an die einschlägigen sozialwissenschaftlichen Konzeptionen von Gemeinschaft (sensu Tönnies, 1912) bzw. Vergemeinschaftung (sensu Weber, 1980) anschliessen lassen: Im Fall der Online-Communities liegt die fehlende Anschliessbarkeit vor allem darin begründet, dass die traditionellen Gemeinschaftskonzepte an einer Vorstellung von ko-präsenten Akteuren angelehnt sind - einer kommunikativen Bedingung also, die für mediatisierte Gemeinschaften wie z.B. Online-LeserInnencommunities nicht vorausgesetzt werden kann. Bei den Diskursgemeinschaften liegt der fehlende Konnex hauptsächlich in der Inkompatibilität der ihr zugrundeliegenden theoretischen Prämissen begründet. Der Begriff ist in der germanistischen ${ }^{1}$ Diskurslinguistik nach Spitzmüller und Warnke (2011, S. 181-182) als Gemengelage komponiert, die am soziolinguistischen Konzept der „Sprachgemeinschaft”, am Ausdruck der „Diskursgesellschaften” (,société de discours") nach Foucault (1971, S. 38-47) und an Max Webers (1980, S. 21-23) Notio der Vergemeinschaftung angelehnt ist. Eine erste Friktion ergibt sich in dieser Begriffskomposition durch die Koppelung der Diskursanalyse an die handlungstheoretisch ausgerichtete Theorie Max Webers, handelt es sich bei Webers (1980) theoretischen Grundbausteinen der „Handlung”, der „Subjektivität” und der „Intentionalität” doch um Konzepte, deren Überwindung als einer der zentralen Antreiber hinter der Diskursanalyse nach Foucault aufgefasst werden kann (vgl. z.B. Gehring, 2012) ${ }^{2}$.

1 Einen Überblick über die Konzeptualisierung von Diskursgemeinschaften in der romanistischen Diskurslinguistik und weiteren sachverwandten Disziplinen bietet Richter (2015, S. 39-64).

2 Vgl. dagegen zur potenziellen Kompatibilität von diskursanalytischen und handlungstheoretischen Ansätzen Spieß (2018). 
Eine weitere Aporie betrifft das ungeklärte Verhältnis bzw. die begriffliche Engführung von Diskursgemeinschaften mit diskursiven Positionen: Es ist unklar, ob die Zugehörigkeit zu einer Diskursgemeinschaft mit der Positionierung im Diskurs zusammenfällt (wie z.B. bei Fix, 2012) oder ob die Zugehörigkeit - unabhängig von der diskursiven Positionierung - allein durch die Teilhabe am Diskurs erfolgt und sämtliche diskursiven Positionen dann jeweils in summa eine gemeinsame Diskursgemeinschaft bilden (vgl. z.B. Knoblauch, 2001, S. 215-216). Angesichts dieser dichotomischen Unbestimmtheit stellt sich nicht zuletzt die Frage, welchen Mehrwert die kategoriale Zuschreibung zu einer Gemeinschaft überhaupt hat, wenn sie unter allen Umständen entweder mit der Positionierung in oder mit der Teilnahme an einem Diskurs zusammenfällt. Ebenso offen bleibt in den einschlägigen diskurslinguistischen Studien, nach welchem Kriterium die Zugehörigkeit zu einer Diskursgemeinschaft bestimmt wird und wer über diese Zugehörigkeit befindet - die Mitglieder der Gemeinschaft oder die analysierenden Forscher*innen?

Vor diesem Hintergrund stehen in der vorliegenden explorativen Studie am Beispiel ausgewählter Leser*innenkommentare des Newsportals watson.ch Vergemeinschaftungspraktiken in Online-Communities und deren theoretische Anschlussfähigkeit im Fokus. Anstatt die Online-Community einfach als Diskursgemeinschaft (oder als Konglomerat aus Diskursgemeinschaften) vorauszusetzen, wird das Vorgehen im Folgenden umgekehrt und das empirische Material ${ }^{3}$ zuerst nach Indizien für Vergemeinschaftung durchleuchtet. Die theoretische Grundlage bilden dabei die zwei einschlägigen Vergemeinschaftungsmerkmale nach Max Weber (1980): traditionale und affektive Handlungen. In Kapitel 3 wird ein Lösungsvorschlag skizziert, wie die Systemtheorie nach Luhmann einen deskriptiven Rahmen für die beobachteten Vergemeinschaftungsindizien in Kommentarforen anbieten und die in dieser Einleitung skizzierten Aporien zwischen verschiedenen Vorstellungen von Gemeinschaft auflösen kann.

\section{Indizien von Vergemeinschaftung in Kommentarforen}

Im vorliegenden Kapitel werden Hinweise auf traditionale (2.1) und affektive (2.2) Handlungen in Online-Kommentaren als klassische Indikatoren der Vergemeinschaftung nach Weber (1980, S. 21) den Ausgangspunkt bilden, um einen sozialwissenschaftlich anschlussfähigen Zugriff auf die linguistische Untersuchung von Online-Gemeinschaften zu schaffen. Die Kommentarsequenz $\left[\mathrm{K} 1_{0-5}\right]^{4}$ (vgl. Abb. 1) soll im Folgenden als illustratives Beispiel dienen, in dem die hier

3 Die rund 2500 Kommentare wurden zwischen 2017 und 2020 im Rahmen des Dissertationsprojektes „Die Kommentare der Gesellschaft. Eine textlinguistische Untersuchung der Funktion von Leserkommentaren zwischen Ethnomethodologie und Systemtheorie" in einer Art teilnehmend beobachtender Online-Feldstudie gesammelt (vgl. Naef, 2021).

4 Die Kommentare sind mit der Signatur [Kn] versehen und fortlaufend nummeriert. Kommentarsequenzen bestehen jeweils aus einem Mutterkommentar $\left[\mathrm{Kn}_{0}\right]$ und Reaktionen darauf, die 
im Fokus stehenden Indikatoren von Vergemeinschaftung zusammen auf engstem Textraum auftreten.
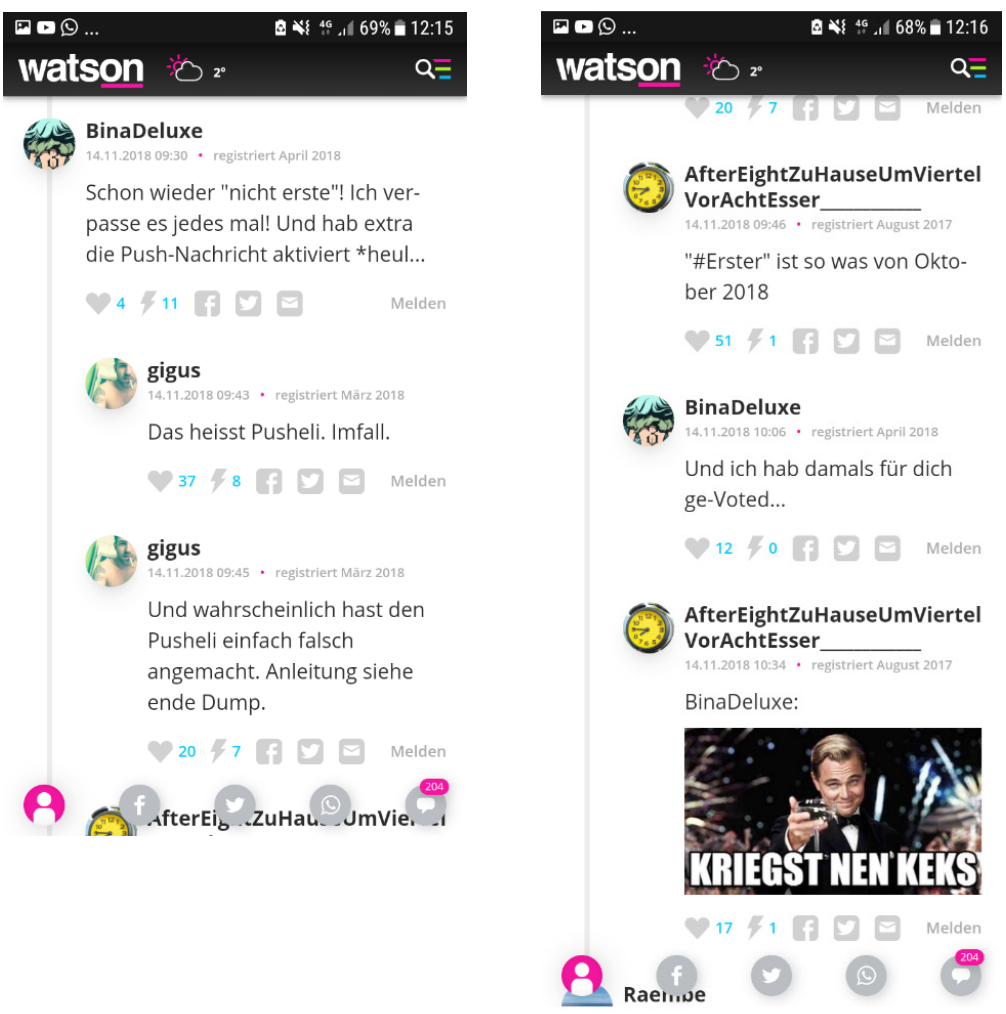

Abbildung 1: Vergemeinschaftungsindizien $\left[\mathrm{K}_{0-5}\right]$

\subsection{Erster!: Von Traditionen und Codes}

Beim Ritual, das hier hinsichtlich seines traditionalen Charakters im Fokus steht, handelt es sich um einen zeitlichen Wettlauf, den ersten Kommentar unter einem Artikel - in diesem Fall dem wöchentlich erscheinenden Picdump - zu verfassen. Dieser Kommentar soll dabei nichts Weiteres als das performative Erste ${ }^{*}$ ! (oder: \#erste ${ }^{*}$ ) beinhalten, der zweite Kommentar dann entsprechend Zweite ${ }^{*}$ ! , usf. (vgl. auch den scherzhaften Kommentar 200.!! [K2] an Position 203). Im Beispiel $\left[\mathrm{K}_{0}\right]$ wird mit der Negation "nicht erste!" meta-performativ auf diese Praktik angespielt (vgl. Abb. 1). Dass es sich dabei um eine Praktik mit wiederkehrendem (und in diesem Sinne traditionalem) Charakter handelt, wird gleich zweimal durch

gemäss ihrer Reihenfolge bezüglich des Mutterkommentars fortlaufend tiefgestellt nummeriert sind. Im Quellenverzeichnis finden sich die Links zu den Kommentaren. 
Temporaladverbien angezeigt (Schon wieder $\left[\mathrm{K}_{0} 1_{0}\right.$; jedes mal $\left.\left[\mathrm{K} 1_{0}\right]\right)$. Im Gegensatz zur Kommentarsektion in anderen Rubriken des Newsportals, in denen sich die Sinngebungsprozesse jeweils nur im Rahmen des prätextuellen Artikels erschliessen, wird das Picdump-Forum dadurch zu einem sozialen Ort konstituiert, an den man zurückkehrt. Durch diese sprachlich erzeugte prospektive Sozialität, findet im Picdump-Forum ein für Newsportale unüblicher kategorialer Sprung von einer situativen, am jeweiligen Artikel orientierten Vergemeinschaftung hin zu einer perdurativen Gemeinschaft statt. Dieser perdurative Charakter ermöglicht es, dass für die Lesbarkeit des Picdump-Forums eine Vertrautheit als Lesbarkeitsquelle (vgl. Hausendorf, Kesselheim, Kato, \& Breitenholz, 2017, S. 96-105) vorausgesetzt werden kann, aus der ausschliesslich eingeweihte Mitglieder der Community schöpfen können. Die Community hat dadurch einen tradierten Insider-Code entwickelt, deren Inklusions- bzw. Exklusionscharakter nicht selten an die textuelle Oberfläche tritt, wie in $\left[\mathrm{K} 1_{1}\right]$ die Reaktion Das heisst Pusheli.

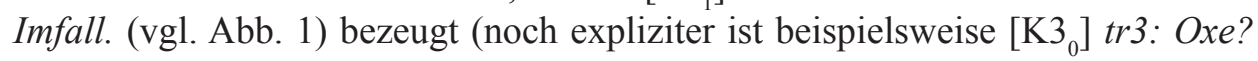
$\left[\mathrm{K}_{1}\right]$ Grubinski: Noch nie Cutenews gelesen? $)^{5}$. Die tradierten Codes werden dadurch zum konstitutiven Wissensrepertoire für die Zugehörigkeit zur Community stilisiert.

\subsection{KRIEGST NEN KEKS: Von Herzen und Blitzen}

Ein zweites essenzielles Kriterium der Vergemeinschaftung ist nach Weber (1980, S. 21) die ,affektuelle (...) Zusammengehörigkeit” bzw. die ,affektuelle oder emotionale (...) Grundlage" (S. 22), auf der Vergemeinschaftung beruht. Im Beispiel (vgl. Abb. 1) kommt diese affektuelle Komponente multimodal mittels Sprache (Und ich habe damals für dich ge-Voted $\left.\left[\mathrm{K}_{4}\right]\right)^{6}$, Sprach-Bild-Kombination (KRIEGST NEN KEKS Meme $\left[\mathrm{K}_{5}\right]$ ) und den ikonischen Textbausteinen „Herz” [Zustimmung] und „Blitz” [Ablehnung] zum Ausdruck. Die sprachlichen Affektbekundungen gehen häufig einher mit der in 2.1 beschriebenen Konstitution des Forums als Ort mit sozialer Perduration. Die visuelle Komponente der Sprach-Bild-Kombinationen dient insbesondere bei affektuell aufgeladenen Posts als Gesten- und Mimiksubstitut. Spätestens bei der affektuellen Ausprägung der ikonischen Textbausteine „Herz” und „Blitz” gelangt man jedoch an einen Punkt, wo die methodische Stringenz bei der Übertragung von Webers Merkmalen der Vergemeinschaftung auf Online-Communities zu bröckeln beginnt. Einerseits offenbart sich in diesem Zusammenhang, dass es sich bei diesen affektuellen Handlungen stets um öffentliche mediale Inszenierungen handelt, und es nicht möglich

5 Zu den Insider-Codes der Watson-Community gehören beispielsweise die Pluralform „Otten” statt „Otter”, „Pusheli” statt „Push”, „Oxe” statt „Ochse”.

${ }^{6}$ Die Aussage bezieht sich auf eine inzwischen nicht mehr existente Praktik des Picumps, jeweils per Abstimmung zu entscheiden, wer in der Folgewoche „Erster!” (vgl. Kap. 2.1) sein darf. 
ist, die funktionalen Fliehkräfte dieser medialen Öffentlichkeit aussen vor zu lassen. Andrerseits verfällt man bei der affektuellen Analyse der Kommentare dem Intentionalitätsdilemma, da eine handlungstheoretische Rekonstruktion darauf angewiesen ist, die affektuelle Absicht hinter den Bekundungen nachzuweisen. Um dieses methodische Dilemma, das in den Literaturwissenschaften bekanntlich unter dem Stichwort der „Autorintention” Eingang in die philologische Theorie gefunden hat, zu umschiffen und auf die Kommentare in ihrer medialen Einbettung zugreifen zu können, wird im folgenden Kapitel um eine systemtheoretische Perspektive erweitert.

Als Zwischenfazit lässt sich vorerst festhalten, dass die Vergemeinschaftungspraktiken nach Max Weber, die sich bei dieser Form von Online-Communities offenbaren, in einem tendenziell disparaten Verhältnis zu dem stehen, was gemeinhin als Konstituierungsverfahren von Diskursgemeinschaften - z.B. die Schaffung eines gesellschaftspolitisch positionierten Wirs - verstanden wird. Auch diesem Umstand soll in der systemtheoretischen Beschreibung mit einem Erklärungsversuch Rechnung getragen werden.

\section{Hinweise auf soziale Funktionssysteme}

Im Folgenden wird skizziert, wie die Systemtheorie nach Niklas Luhmann einen deskriptiven Rahmen bieten könnte, um einerseits die in Kapitel 2 beobachteten Phänomene kommunikationstheoretisch anschlussfähig zu verorten und andrerseits die Unvereinbarkeit zwischen diskursanalytischen und soziologischen Vorstellungen von Gemeinschaft zu überwinden. In Kapitel 3.1 werden die kommunikationsspezifischen Bedingungen der Kommentarforen mit dem Luhmannschen Kommunikationsverständnis angegangen, um einen theoretischen Rahmen für die bisher angedeuteten Probleme zu entwerfen, welche sich für die Vergemeinschaftung unter den spezifischen Bedingungen von Online-Kommentarforen im massenmedialen Kontext ergeben. In 3.2 wird die bei Weber angelegte Gegenüberstellung von Gemeinschaft und Gesellschaft überführt in die Luhmannsche Unterscheidung dreier Ebenen der sozialen Systembildung: Interaktion, Organisation und Gesellschaft. Diese Ebenen dienen als ordnender Rahmen, um das polyfunktionale Geflecht aus Community, Medium und Öffentlichkeit, das sich in Kommentarforen entfaltet, zu entwirren, und es in analytisches Potential zur Erklärung empirischer Phänomene umzumünzen.

\subsection{Kommunikation als Unwahrscheinlichkeit}

Ein systemtheoretischer Zugriff auf textuelle Online-Kommunikation kann die in Kap. 2.2 thematisierten Unzulänglichkeiten handlungstheoretischer Ansätze bezüglich Intentionalität insofern überwinden, als sie nicht von Akteuren als konstitutivem Prinzip des Sozialen ausgeht, sondern an seine Stelle das Primat der Kommunikation setzt: die soziale Welt konstituiert sich demnach ausschliesslich 
über Kommunikation und ist nur über sie beobachtbar. Für die skizzierten Problembereiche des Akteurs und der Intention bedeutet dies, dass sie nur in dem Grad für den analytischen Zugriff von Bedeutung sind, wie sie in ihrer kommunikativen Veräusserung empirisch beobachtbar sind. Kommunikation ist dabei aber keinesfalls als Selbstläufer zu verstehen. Im Gegenteil: Kommunikation wird in der Systemtheorie nach Luhmann als Unwahrscheinlichkeit verstanden (vgl. Luhmann, 1981). Das Zustandekommen von Kommunikation ist demnach stets vor drei ihr inhärenten und selbsterschaffene Probleme gestellt, die es immer wieder von Neuem zu lösen gilt: „Verstehen”, „Erreichen” und „Erfolg” (vgl. Luhmann, 1981, S. 26-27). Im Fall von Online-Leser*innenkommentaren lassen sich die iterative Überwindung der selbstgeschaffenen kommunikativen Unwahrscheinlichkeiten und die daraus entstehenden Lösungsmuster anhand dieser drei Problempfeiler folgendermassen skizzieren:

Das „Verstehen” von Kommunikation ${ }^{7}$ ist im online-massenmedialen Kontext in mehrfacher Hinsicht problematisch. Einerseits gehören die Kommunikate aufgrund ihres hohen Grads an Voraussetzungsreichtum bezüglich Wissen (z.B. über Vorgänge in weit entfernten Regionen) im Kontext einer längst angelaufenen, selbstbezüglichen Kommunikationskette zu den anspruchsvolleren Textsorten. Leserinnenkommentare können insofern als Antwort auf das Problem des Verstehens gesehen werden, als sie Lektüremöglichkeiten von Artikeln reflexiv darstellen, sie damit potentiell einschränken. Ähnlich einer Publikumsinszenierung im Fernsehen (vgl. Hausendorf, 2001) zeigen sie dadurch sinnvolle Reaktionen und mögliche kommunikative Anschlüsse an das Gelesene auf. Dabei wird häufig auch das Nichtverstehen eines Artikels zum Thema (vgl. z.B. [K4] What the fuck did I just read? (:)). Angesichts der asymmetrischen Struktur der massenmedialen Kommunikation, die gewissermassen einfach ins Blaue hinaussendet, bietet das Forum als Form der Rückkommunikation (vgl. Luhmann, 2017, S. 26) für das Medium die Möglichkeit, zu beobachten, wie und ob die ausgesandte Kommunikation verstanden wird. Eine Sonderrolle kommt in diesem Punkt den in Kap. 2.2 beschriebenen Codes (Otten, Pusheli, usf.) zu, welche - entgegen dem medialen Selbstverständnis als Informationsvermittlerin - gezielt auf Nichtverstehen angelegt sind. Durch die Verschiebung der Betrachtungsperspektive auf Kommunikations-spezifische Fragestellungen lassen sich diesbezüglich nun mindestens zwei neuartige Aspekte ergänzen: 1) Es lässt sich feststellen, dass Kommunikation in dem Masse gemeinschaftsstiftend ist, wie sie als verständnisbasierte Mechanismen der Inklusion/Exklusion operieren (ähnlich, wie wenn gegenüber Dritten gezielt eine Sprache gesprochen wird, welche diese nicht verstehen). In Gemeinschaften wird also - entgegen gemeinläufigen Vorstellungen von Traditionen - nicht

7 In der Terminologie der Systemtheorie entspricht „Verstehen” der Unterscheidung von Information und Mitteilung (vgl. Luhmann, 2018a, S. 191-242 und 2018b, S. 107-118). 
einfach Wissen (beispielsweise über die Orthografie von Wörtern) transportiert, sondern das Wissen muss, damit es gemeinschaftsstiftend sein kann, so tradiert werden, dass es als Ein- und Ausschlussmechanismus operieren kann. 2) Dadurch verlieren die Vergemeinschaftungspraktiken bis zu einem gewissen Grad auch ihre Harmlosigkeit. Es wäre übertrieben zu behaupten, dass durch diese Insidercodes in der Kommentarspalte auf watson.ch jemand ernsthaft zu Schaden kommt, jedoch dürfte ein Teil des unifizierenden Potentials dieser Praktiken auch aus dem Wissen schöpfen, dass man - zumindest in der operativen Logik der Massenmedien - mit Insidercodes gegen kooperative Prinzipien der Kommunikation verstösst. Das Gemeinschaftsgefühl entpuppt sich auf Ebene des Verstehens als Resultat der gemeinsamen Teilhabe an einem Akt mit kommunikativem Deliktcharakter.

Einer der zentralen Treiber hinter der strukturellen Ausprägung von Online-Kommentarforen ist das Problem des „Erfolgs” bzw. des „Annehmens” von Kommunikation. Während es in Situationen, die unter der Bedingung der leiblichen Kopräsenz stattfinden, sozial riskant (und unter Androhung physischer Gewalt sogar lebensgefährlich) sein kann, Kommunikation abzulehnen, ist die Ablehnung eines kommunikativen Angebots eines massenmedial operierenden Newsportals risikolos und deshalb sogar der häufigere Fall, als «dass der Empfänger den selektiven Inhalt der Kommunikation (die Information) als Prämisse des eigenen Verhaltens übernimmt» (Luhmann, 1981, S. 26). Online-Communities können in diesem Rahmen als Lösungsroutine auf das Problem des kommunikativen Erfolgs massenmedialer Onlinemedien verstanden werden, weil die Ablehnung von Kommunikation bei zunehmend ausgeprägter Gemeinschaftsbildung registriert und damit sozial riskanter wird (so z.B. in [K5] Wo ist eigentlich Pun?, als eines von zahlreichen Beispielen, in denen die lange Absenz eines Users explizit thematisiert wird).

Das Problem der Erreichbarkeit ist im Falle der massenmedialen Online-Kommunikation zumindest in technischer Hinsicht ein de facto gelöstes Problem. Mit einem geeigneten Empfangsgerät kann die Kommunikation eines beliebigen Online-Mediums an jedem habitablen Punkt der Erde (und darüber hinaus) empfangen werden. Komplizierter wird die Sache, wenn man sich das kommunikative Überangebot an Informationen, das im World Wide Web ohne direkte monetäre Kosten zur Verfügung steht, aus Sicht der Rezipient*innen vor Augen führt. Vor diesem Hintergrund tragen Leser-Communities dazu bei, dass die ausgesendeten Kommunikationen eine mehr oder weniger treuen Leserstamm (Stichwort: Leseranbindung) erreicht, welcher nicht nur über Pushnachrichten sondern auch über die Benachrichtigung, wenn jemand auf einen Kommentar reagiert hat, kommunikativ erreicht wird ${ }^{8}$.

8 In diesem Zusammenhang ist es auch wichtig zu erwähnen, dass die drei Probleme gemäss der Kommunikation Luhmann (1981, S. 27) in einem invers interpendenten Verhältnis stehen; d.h., 
Zusammenfassend lässt sich aus systemtheoretischer Perspektive festhalten, dass sich Vergemeinschaftungstendenzen von Online-Communities im Rahmen von Lösungsroutinen der drei kommunikativen Grundprobleme „Erreichen”, „Erfolg” und „Verstehen” beschreiben lassen. Im Gegensatz zum kulturpessimistisch angehauchten Ansatz der posttraditionalen Gemeinschaft (vgl. Hitzler, Honer, \& Pfadenhauer, 2008), wo die kommunikative Ausgestaltung dieser neuen Sozialformen als Ausdruck einer individualisierten und emotionsentbundenen Kultur verstanden wird, lässt sich aus systemtheoretischer Perspektive eher darüber staunen, dass unter den Bedingungen der Online-Kommunikation soziale Gefüge mit Gemeinschaftscharakter angesichts der steten Möglichkeit, Kommunikation abzulehnen, überhaupt möglich sind.

\subsection{Ebenen sozialer Systembildung}

In der Systemtheorie wird die auf Tönnies (1912) und Weber (1980) zurückzuführende dichotomische Gegenüberstellung von Gemeinschaft und Gesellschaft zugunsten von drei ineinander verschränkten Ebenen der sozialen Systembildung aufgelöst: Interaktion, Organisation und Gesellschaft (vgl. Luhmann 1975)9 . Der Unterschied zwischen den drei Ebenen liegt in den kommunikativen Voraussetzungen, durch die sich die Ebenen konstituieren: Das konstitutive Element der Interaktion ist dabei die Bedingung der „wechselseitigen Wahrnehmung” (vgl. Luhmann, 1975, S. 10-11), für Organisationen die „Mitgliedschaft” (S. 12-13) und für die Ebene der Gesellschaft die „Erreichbarkeit” (S. 11-12).

Im Falle von Online-Kommentaren lassen sich über diese Ebenendifferenzierung folgende integrative Beschreibungsperspektiven andenken: Eine erste bemerkenswerte Beobachtung besteht aus systemtheoretischer Perspektive darin, dass es sich bei Online-Communities aufgrund der fehlenden Wahrnehmungswahrnehmung „,nicht” um „Interaktion” handelt (vgl. Esposito, 1995). Aus methodologischer Perspektive bedeutet das, dass die komparative Übertragung von auf Ko-Präsenz beruhenden Kriterien der Vergemeinschaftung ${ }^{10}$ auf Online-Kommunikation, wie dies beispielsweise bei Hitzler et al. (2008) geschieht, kritisch zu betrachten ist.

dass die Lösung des einen Problems mit der Verstärkung der anderen beiden Probleme einhergeht. Beim Problem der Erreichbarkeit lässt sich das sehr anschaulich anhand einer simulierten Maximierung der Erreichbarkeit demonstrieren, indem man eine zufällig ausgewählte Website öffnet [vgl. z.B. Q2]. Die Wahrscheinlichkeit, dass man die geöffnete Seite (nur schon sprachlich) versteht, ist dabei verschwindend klein.

9 Vgl. als Einführung und zur kritischen Diskussion dieser drei Ebenen Heintz und Tyrell (2015).

${ }^{10}$ Vgl. zur überholten Vorstellung einer auf Ko-Präsenz beruhenden Gesellschaft Dickel (2020). 
Aus systemtheoretischer Perspektive lassen sich viele Phänomene von Online-Leser*innenkommentaren aufschlüsseln, wenn sie als Form von Kommunikation auf der sozialen Systemebene der Organisation verstanden werden. Diese auf den ersten Blick kontraintuitive Beobachtung schärft einerseits das empirische Auge für einen vergleichsweise neuen Baustein in den Leserkommentaren von watson.ch in dem das Kriterium der Mitgliedschaft durch die Angabe des Registrationszeitpunktes (vgl. z. B. [K1]; Registriert April 2018) an die textuelle Oberfläche getragen wird, was für die kommunikative Struktur des Forums beispielsweise in Bezug auf den Status eines Beitrags von Bedeutung sein kann (vgl. z.B. Du und Fabian Lehner seid noch nicht lange hier auf watson.ch, oder? [K6 $\left.6_{1}\right]$ ). Die Ebene der Organisation operiert auch als entscheidender Vergleichswert bei der Differenzierung der Foren nach aussen (vgl. Die watson-Kommentarspalte hat nun offiziell das Niveau der 20-Minuten Kommentarspalte erreicht $\left.\left[K^{*}\right]^{11}\right)$. Durch das Kriterium der Mitgliedschaft öffnet sich andrerseits die Möglichkeit einer diachronen Vergleichsperspektive von Online-Leserkommentaren und Leserbriefen, welche (unter Angabe von Namen und postalischer Adresse) unter dem Kriterium der Erreichbarkeit operiert haben. Es findet hier also ein sozio-struktureller Ebenensprung statt, der die Leserschaft unterscheidet in eine rezipierende Gesellschaftsöffentlichkeit, die kommunikativ erreichbar ist, und eine in die Organisation integrierte Gruppe mit Mitgliederstatus ${ }^{12}$.

Auf Ebene der Gesellschaft sind Online-Kommentarforen insbesondere hinsichtlich der darin erkennbaren Koppelungseffekte zwischen den Funktionssystemen Massenmedien und Wirtschaft (Stichwort «User als Produkt») von Interesse, die den Rahmen einer linguistischen Analyse aber grundsätzlich übersteigen. Für die Zwecke dieses Artikels ist die Ebene der Gesellschaft deshalb von Bedeutung, weil auf dieser Ebene wieder Phänomene ins Spiel kommen, welche an das Konzept der Diskursgemeinschaften anschliessbar sind. Aus systemtheoretischer Perspektive besteht eine primäre Funktion der Massenmedien in der reflexiven Herstellung einer öffentlichen Meinung (vgl. Luhmann, 2017, S. 128). Erst dadurch lässt sich die textfunktionale Struktur von Kommentaren, welche aus textlinguistischer Perspektive in einer Dominantsetzung der emotiven Funktion, welche nach Jakobson (2016, S. 89-90) «die Haltung des Sprechers zum Gesprochenen unmittelbar zum Ausdruck [bringt]», sinnvoll in ihrer Wirkungsentfaltung aufschlüsseln. Die aus den Texten rekonstruierbaren musterhaften Diskurspositionen zeigen also vorerst keine Hinweise auf Gemeinschaft, sondern sie sind die mediale Inszenierung einer öffentlichen Meinung, an der sich das Publikationsmedium wiederum thematisch ausrichtet. Aus systemtheoretischer Perspektive lässt sich

\footnotetext{
${ }^{11}$ Der Kommentar ist online nicht mehr verfügbar, liegt dem Verfasser aber als Screenshot vor.

${ }_{12}$ Dieser Aspekt ist auch für die damit einhergehenden Effekte der Leseranbindung nicht unbedeutend (vgl. Kap. 3.1)
} 
also konstatieren, dass es sich bei Diskursgemeinschaften nicht um Gemeinschaften, sondern um den Vollzug kommunikativer Strukturen des Funktionssystems der Massenmedien handelt. Wenn diese Positionen dann den Ausgangspunkt für soziale Vergemeinschaftungsphänomene bilden, entkoppelt sich die Struktur von diskursiven Phänomenen der Gesellschaftsebene und manifestiert sich wiederum als organisationale Kommunikation. Deshalb ist es tendenziell ausgeschlossen, dass sich in nicht-organisational strukturierten Online-Communities Gemeinschaften herausbilden. Zusammenfassend lässt sich also sagen, dass sich Diskurspositionen in Organisationen sedimentieren, während das Habitat des Diskurses die Gesellschaft ist.

\section{References}

Dickel, S. (2020). Gesellschaft funktioniert auch ohne anwesende Körper. Die Krise der Interaktion und die Routinen mediatisierter Sozialität. In M. Volkmer, \& K. Werner (Eds.), Die Corona-Gesellschaft (pp. 79-86). Bielefeld: Transcript. DOI:10.14361/9783839454329-008.

Foucault, M. (1971). L'ordre du discours. Paris: Gallimard.

Esposito, E. (1995). Interaktion, Interaktivität und die Personalisierung der Massenmedien. Soziale Systeme, 2, 225-260.

Fix, U. (2012). Leserbriefe - die mediale Konstruktion von Diskursgemeinschaften. In C. Grösslinger, G. Held, \& H. Stöckl (Eds.), Pressetextsorten jenseits der „News“ (pp. 139-156). Frankfurt am Main: Peter Lang Verlag.

Gehring, P. (2012). Abseits des Akteurs-Subjekts. In R. Keller, W. Schneider, \& W. Viehöver (Eds.), Diskurs - Macht - Subjekt (pp. 21-33). Wiebaden: Verlag für Sozialwissenschaften. DOI: 10.1007/978-3-531-93108-1_2.

Hausendorf, H. (2001). Warum wir im Fernsehen so häufig begrüßt und angeredet werden: eine exemplarische Studie am Beispiel der Sendung mit der Maus. In T. Sutter, M. Tilmann, \& M. Charlton (Eds.), Massenkommunikation, Interaktion und soziales Handeln (pp. 185-213). Opladen: Westdeutscher Verlag.

Hausendorf, H., Kesselheim, W., Kato, H., \& Breitenholz, M. (2017). Textkommunikation: ein textlinguistischer Neuansatz zur Theorie und Empirie der Kommunikation mit und durch Schrift. Berlin: De Gruyter. DOI:10.1515/9783110557435.

Heintz, B., \& Tyrell, H. (Eds.) (2015). Interaktion-Organisation-Gesellschaft revisited: Anwendungen, Erweiterungen, Alternativen. Stuttgart: Lucius \& Licius. DOI:10.1515/9783110509243.

Hitzler, R. \& Honer, A., \& Pfadenhauer, M. (Eds.) (2008). Posttraditionale Gemeinschaften. Wiesbaden: Verlag für Sozialwissenschaften. DOI: 10.1007/978-3-531-91780-1.

Jakobson, R., (2016). Linguistik und Poetik. In E. Holenstein, \& T. Schelbert (Eds.) Roman Jakobson. Poetik. Ausgewählte Aufsätze 1921-1971 (pp. 83-121). Frankfurt am Main: Suhrkamp.

Knoblauch, H. (2001). Diskurs, Kommunikation und Wissensoziologie. In R. Keller, A. Hirseland, W. Schneider, \& W. Viehöver (Eds.) Handbuch Sozialwissenschaftliche Diskursanalyse (pp. 207-223). Wiesbaden: Verlag für Sozialwissenschaften. DOI: 10.1007/978-3-322-99906-1_8.

Luhmann, N. (1975). Interaktion - Organisation - Gesellschaft. In N. Luhmann (Ed.), Soziologische Aufklärung 2: Aufsätze zur Theorie der Gesellschaft (pp. 9-20). Opladen: Westdeutscher Verlag.

Luhmann, N. (1981). Die Unwahrscheinlichkeit der Kommunikation. In N. Luhmann (Ed.), Soziologische Aufklärung 3: Soziales System, Gesellschaft, Organisation (pp. 25-34). Opladen: Westdeutscher Verlag.

Luhmann, N. (2017). Die Realität der Massenmedien. Wiesbaden: Springer. 
Luhmann, N. (2018a). Soziale Systeme. Grundriss einer allgemeinen Theorie. Frankfurt am Main: Suhrkamp.

Luhmann, N. (2018b). Was ist Kommunikation? In N. Luhmann (Ed.), Soziologische Aufklärung 6: Die Soziologie und der Mensch (pp. 25-34). Wiesbaden: Springer.

Naef, M. (2021). Die Kommentare der Gesellschaft. Eine textlinguistische Untersuchung der Funktion von online Leserkommentaren zwischen Ethnomethodologie und Systemtheorie. Manuscript in preparation.

Richter, J. (2015). Die Konstruktion von Reputation: Verweise auf Ferdinand de Saussure in der romanistischen Sprachwissenschaft. Bielefeld: Transcript. DOI: 10.14361/9783839433164-001.

Spieß, C. (2018). Diskurs und Handlung. In I. H. Warnke (Ed.), Handbuch Diskurs. Berlin, Boston: De Gruyter. DOI: 10.1515/9783110296075-014.

Spitzmüller, J., \& Warnke, I. H. (2011). Diskurslinguistik: Eine Einführung in Theorien und Methoden der transtextuellen Sprachanalyse. Berlin, Boston: De Gruyter. DOI: 10.1515/9783110229967.

Tönnies, F. (1912). Gemeinschaft und Gesellschaft. Grundbegriffe der reinen Soziologie. Zweite erheblich veränderte und vermehrte Auflage. Berlin: Curtius.

Weber, M. (1980). Wirtschaft und Gesellschaft. Grundriß der verstehenden Soziologie. Tübingen: Mohr Siebeck.

\section{Primary Sources retrieved December 8, 2020}

K1-2 https://www.watson.ch/!721056798

K3 https://www.watson.ch/quiz/tv/298301442-wer-wird-millionaer-bei-dieser-frage-muessenzwei-kandidaten-aufgeben

K4 https:/www.watson.ch/dumm\%20gelaufen/england/553238928-in-england-hat-sich-geradedas-schlimmste-tinder-date-ever-ereignet-es-hat-so-geendet?

K5 https:/www.watson.ch/spass/picdump/789552896-zeit-fuer-den-pidcump-arbeiten-koennenwir-noch-spaeter-oder-auch-nicht?ub\&ss=1\&ut=1\&sp\&utm_av=B

K6 https://www.watson.ch/sport/wissen/126307563-fussball-das-sind-die-besten-passspieler-derwelt\#discussion_126307563

Q1 https://www.watson.ch/u/commenting_rules

Q2 https://www.whatsmyip.org/random-website-machine/ 